\title{
Imaging Intracellular Signaling Using Two-Photon Fluorescent Lifetime Imaging Microscopy
}

\author{
Ryohei Yasuda
}

The recent development of Förster resonance energy transfer (FRET) sensors and FRET imaging techniques permits visualization of the dynamics of intracellular signaling events with high spatiotemporal resolution. In particular, fluorescence lifetime imaging in combination with two-photon laser-scanning microscopy (two-photon fluorescence lifetime imaging microscopy [2pFLIM]) is a powerful tool to monitor signaling events in small subcellular compartments in thick tissue. This article provides practical guidelines for quantitative imaging of intracellular signaling using 2pFLIM.

Förster resonance energy transfer (FRET) is the process of nonradiative energy transfer from an excited donor fluorophore to an acceptor fluorophore through dipole-dipole interactions. The efficiency of FRET strongly depends on the distance between the donor and the acceptor (Förster 1993; Lakowicz 2006), making this phenomenon a useful tool for monitoring interactions between proteins that are fused to fluorophores (Miyawaki 2003). Because FRET increases acceptor fluorescence and decreases donor fluorescence, the ratio between the fluorescence intensities in the donor and the acceptor emission wavelengths is often used to measure FRET (Wallrabe and Periasamy 2005). Alternatively, the fluorescence lifetime of the donor, which is the time between the excitation of the fluorophore and emission of a photon, can be used as a readout of FRET because the fluorescence lifetime shortens as FRET efficiency increases (Wallrabe and Periasamy 2005; Lakowicz 2006; Yasuda 2006). There are several advantages to using fluorescence lifetime imaging microscopy (FLIM) compared with ratiometric imaging. First, the signal is independent of the relative concentration of the donor and the acceptor. Second, the signal is relatively independent of light scattering by the tissue compared with ratiometric imaging, which is affected by the wavelength dependency of light scattering. Third, when multiple populations with different FRET efficiency coexist, the fluorescence decay curve becomes multiexponential, and one can deconvolve each component by fitting the curve (Lakowicz 2006). FLIM has been combined with two-photon laser-scanning microscopy (2pLSM) to image samples in light-scattering tissue. This technique has enabled the measurement of signaling events in small neuronal compartments in brain slices (Yasuda 2006).

\section{TWO-PHOTON FLUORESCENCE LIFETIME IMAGING SETUP}

There are several methods for imaging fluorescence lifetime (Lakowicz 2006). The time-correlated single-photon-counting (TCSPC) method in combination with 2pLSM is optimal for imaging small

Adapted from Imaging: A Laboratory Manual (ed. Yuste). CSHL Press, Cold Spring Harbor, NY, USA, 2010.

(c) 2012 Cold Spring Harbor Laboratory Press

Cite this article as Cold Spring Harb Protoc; 2012; doi:10.1101/pdb.top072090 
R. Yasuda
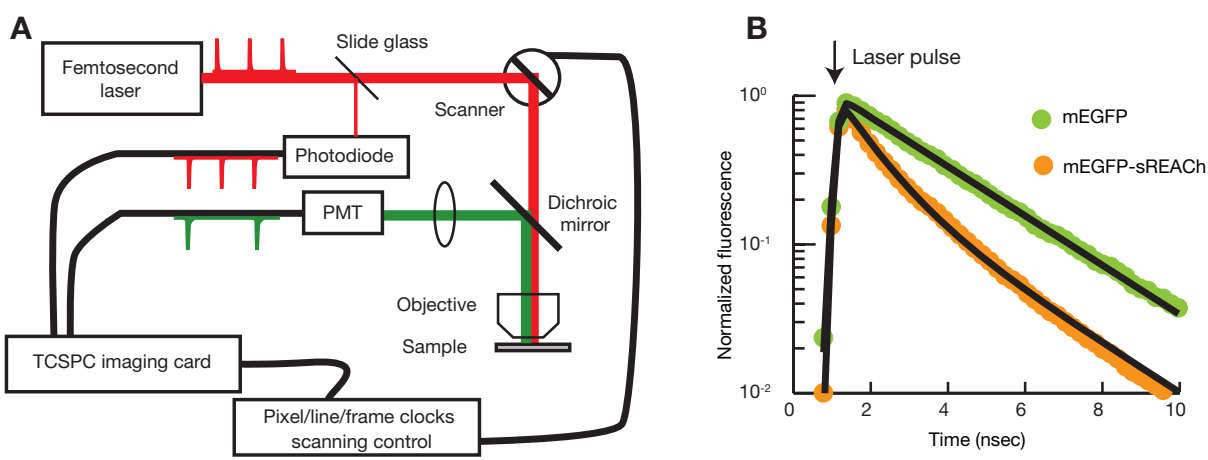

FIGURE 1. Two-photon fluorescence lifetime imaging. (A) Schematic of a 2pFLIM setup. TCSPC, time-correlated singlephoton counting; PMT, photomultiplier tube. (B) Semilog plot of a fluorescence decay curve of monomeric enhanced green fluorescent protein (meGFP) and meGFP-sREACh (superresonance energy-accepting chromoprotein) tandem. meGFP is enhanced green fluorescent protein (eGFP) with the monomeric mutation (A206K) (Zacharias et al. 2002), and sREACh is a variant of nonradiative yellow fluorescent protein (YFP) (Murakoshi et al. 2004). The curves are fitted with Equation 1 for meGFP and Equation 2 for meGFP-sREACh tandem. The curve of meGFP-sREACh shows more than one fluorescence lifetime, presumably corresponding to the fraction of meGFP with folded and unfolded sREACh.

subcellular compartments in light-scattering tissue (Yasuda 2006). TCSPC measures the time that has elapsed between the arrival of a single photon detection pulse and the next laser pulse (Lakowicz 2006). As 2pLSM uses a pulsed laser, it is relatively simple to combine TCSPC with 2pLSM by modifying standard 2pLSM as follows (Yasuda et al. 2006; Murakoshi et al. 2008) (see Fig. 1A).

1. Install TCSPC imaging PCI card (such as PSC-150, Becker \& Hickl GmbH).

2. Use a PMT with low transit-time spread $(<0.2 \mathrm{nsec})$ and single photon-counting sensitivity (such as H-7422P, Hamamatsu).

3. Detect the laser pulses reflected by a slide glass using a fast photodiode (such as FDS010, Thorlabs). Some commercial lasers have a built-in fast photodiode output.

4. Feed the photon signal from the PMT, the laser pulse signal from the photodiode and pixels, and line and frame clocks synchronized with scanning into the TCSPC card.

\section{ANALYSIS OF FLUORESCENCE LIFETIME IMAGES}

Fitting Fluorescence Lifetime Curve

The fluorescence from a fluorophore following a short laser pulse typically decays exponentially (Fig. 1B). Thus, the fluorescence lifetime of the free donor $\tau_{\mathrm{D}}$ can be measured by fitting the fluorescence decay curve with an exponential function convolved with the Gaussian pulse response function of the system (Fig. 1B):

$$
H\left(t, t_{0}, \tau_{\mathrm{D}}, \tau_{\mathrm{G}}\right)=\frac{1}{2} \exp \left(\frac{\tau_{\mathrm{G}}^{2}}{2 \tau_{\mathrm{D}}}-\frac{t-t_{0}}{\tau_{\mathrm{D}}}\right) \operatorname{erf}\left(\frac{\tau_{\mathrm{G}}^{2}-\tau_{\mathrm{D}}\left(t-t_{0}\right)}{\sqrt{2 \tau_{\mathrm{D}} \tau_{\mathrm{G}}}}\right),
$$

where $\tau_{\mathrm{G}}$ is the width of the Gaussian pulse response function, $t_{0}$ is the time offset, and erf is the error function (Fig. 1B). When the free donor and the donor bound to an acceptor coexist, the fluorescence decays according to a double exponential, a mixture of the free donor and the donor bound to the acceptor. Thus, the fraction of the donor bound to the acceptor can be calculated by fitting the fluorescence decay curve with the double exponential curve,

$$
F(t)=F_{0}\left[P_{\mathrm{D}} \cdot H\left(t, t_{0}, \tau_{\mathrm{D}}, \tau_{\mathrm{G}}\right) \cdot P_{\mathrm{AD}} \cdot H\left(t, t_{0}, \tau_{\mathrm{AD}}, \tau_{\mathrm{G}}\right)\right],
$$


where $\tau_{\mathrm{AD}}$ is the fluorescence lifetime of the donor bound with the acceptor, and $P_{\mathrm{D}}$ and $P_{\mathrm{AD}}$ are the fractions of the free donor and the donor bound with the acceptor $\left(P_{\mathrm{D}}+P_{\mathrm{AD}}=1\right)$. To reduce the number of parameters to be fitted, $\tau_{\mathrm{D}}$ can be fixed to the fluorescence lifetime obtained when the free donor is measured separately. It should be noted that some fluorophores, such as enhanced cyan fluorescent protein (eCFP) and its brighter variant Cerulean, have more than one time constant (Tramier et al. 2002; Yasuda et al. 2006). When using these fluorophores as FLIM donors, calculation of the binding fraction will be more complicated.

\section{Visualizing Fluorescence Lifetime Images and Binding Fraction}

Fluorescence lifetime images are in $3 \mathrm{D}(x, y$, and $t)$ or $4 \mathrm{D}(x, y, t$, and $z)$. Thus, the images need to be folded into $2 \mathrm{D}$ to visualize them. One way to visualize fluorescence lifetime images is to code the fluorescence lifetime with color and to code fluorescence intensity with brightness (Yasuda et al. 2006) (Fig. 2). For 4D images, the $z$-axis can be further folded by the commonly used maximum intensity projection, where the maximum intensity in the $z$-axis is assigned for each pixel in an $x-y$ image. Curve-fitting the fluorescence lifetime in each pixel is suboptimal, because it is computationally time consuming and often does not converge well because the number of photons in each pixel is limited. Instead, fluorescence lifetime images can be produced by calculating the mean fluorescence lifetime $\tau_{\mathrm{m}}$ in each pixel as

$$
\tau_{\mathrm{m}}=\langle t\rangle=t_{0}=\frac{\int d t \cdot t F(t)}{\int d t \cdot F(t)}-t_{0}
$$

where $F(t)$ is the fluorescence lifetime decay curve (Yasuda et al. 2006; Murakoshi et al. 2008) (Fig. 2B). The offset $t_{0}$ depends on the optical path length, the cable length, and so on. Its value can be obtained by fitting the fluorescence lifetime curve integrated over the entire image, using either Equation 1 or 2.

When the free donor and the donor bound to an acceptor coexist (Equation 2), $\tau_{\mathrm{m}}$ is given approximately by

$$
\tau_{\mathrm{m}} \sim \frac{\int d t \cdot t\left(P_{\mathrm{D}} e^{-t / \tau_{\mathrm{D}}}+P_{\mathrm{AD}} e^{-t / \tau_{\mathrm{AD}}}\right)}{\int d t \cdot t\left(P_{\mathrm{D}} e^{-t / \tau_{\mathrm{D}}}+P_{\mathrm{AD}} e^{-t / \tau_{\mathrm{AD}}}\right)}=\frac{P_{\mathrm{D}} \tau_{\mathrm{D}}^{2}+P_{\mathrm{AD}} \tau_{\mathrm{A}}^{2}}{P_{\mathrm{D}} \tau_{\mathrm{D}}+P_{\mathrm{AD}} \tau_{\mathrm{AD}}}
$$
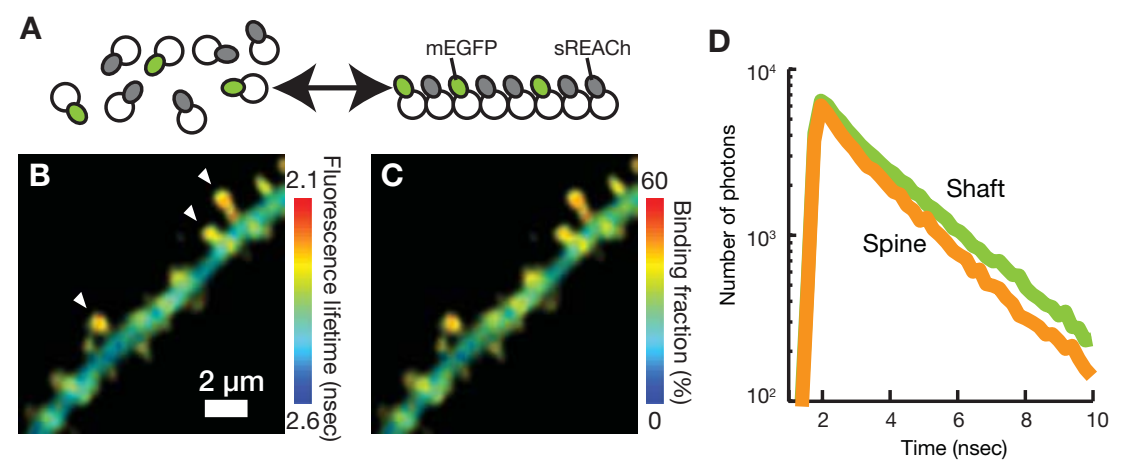

FIGURE 2. Visualization of fluorescence lifetime and binding fraction. (A) Schematic of an actin polymerization sensor. meGFP-actin and sREACh-actin are coexpressed (Murakoshi et al. 2004), and FRET occurs when they form polymeric filaments. $(B)$ A fluorescence lifetime image of a dendritic segment of a neuron expressing the actin polymerization sensor $(A)$ in an organotypic hippocampal slice. The image is produced by using Equation 3. (C) $\mathrm{A}$ binding fraction image calculated from the fluorescence lifetime image $(B)$ using Equation 4 . $(D)$ The fluorescence decay curves in spines (average of three spines indicated with white arrowheads in $B$ ) and the parent dendritic shaft. 
Thus, the binding fraction $\left(P_{\mathrm{AD}}\right.$; see Equation 2) can be obtained by Murakoshi et al. (2008) (Fig. 2C)

$$
P_{\mathrm{AD}}=\frac{\tau_{\mathrm{D}}\left(\tau_{\mathrm{D}}-\tau_{\mathrm{m}}\right)}{\left(\tau_{\mathrm{D}}-\tau_{\mathrm{AD}}\right)\left(\tau_{\mathrm{D}}+\tau_{\mathrm{AD}}-\tau_{\mathrm{m}}\right)}
$$

where $\tau_{\mathrm{D}}$ and $\tau_{\mathrm{AD}}$ can be obtained by fitting the fluorescence decay curve integrated over the entire image (Equation 2). A red-green-blue (RGB) pseudocolor image with the color scheme shown in Figure $2 \mathrm{~A}$ and $\mathrm{B}$ can be generated by using the following scheme.

1. Normalize $\tau_{\mathrm{m}}$ or $P_{\mathrm{AD}}$ so it maps to 0 to 1 range $(h)$.

2. Convert $h$ into RGB colors, such that

$$
\begin{array}{llll}
\mathrm{R}=0 & \mathrm{G}=3 h & \mathrm{~B}=1 & (0 \leq h \leq 1 / 3) \\
\mathrm{R}=3 h-1 & \mathrm{G}=1 & \mathrm{~B}=2-3 h & (1 / 3<h \leq 2 / 3) \\
\mathrm{R}=1 & \mathrm{G}=3-3 h & \mathrm{~B}=0 & (2 / 3<h \leq 1)
\end{array}
$$

3. Normalize the total fluorescence intensity $\left(\int d t \bullet F(t)\right)$ so that it is in the 0 to 1 range, and multiply it to each color component.

The normalization depends on whether FRET increases or decreases with protein activity (compare Fig. 3B with D).

\section{NUMBER OF PIXELS AND SIGNAL LEVEL}

In TCSPC, there is some dead time after each photon detection event, so the maximum photoncounting rate is limited to $\sim 10^{6} \mathrm{~Hz}$. This may limit the temporal resolution of the system. Quantitatively speaking, the noise of the mean fluorescence lifetime $\left(\delta \tau_{\mathrm{m}}\right)$ is related to the number of photons counted (N) (Philip and Carlsson 2003) as

$$
\frac{\delta \tau_{\mathrm{m}}}{\tau_{\mathrm{m}}} \sim \frac{1}{\sqrt{N}} .
$$
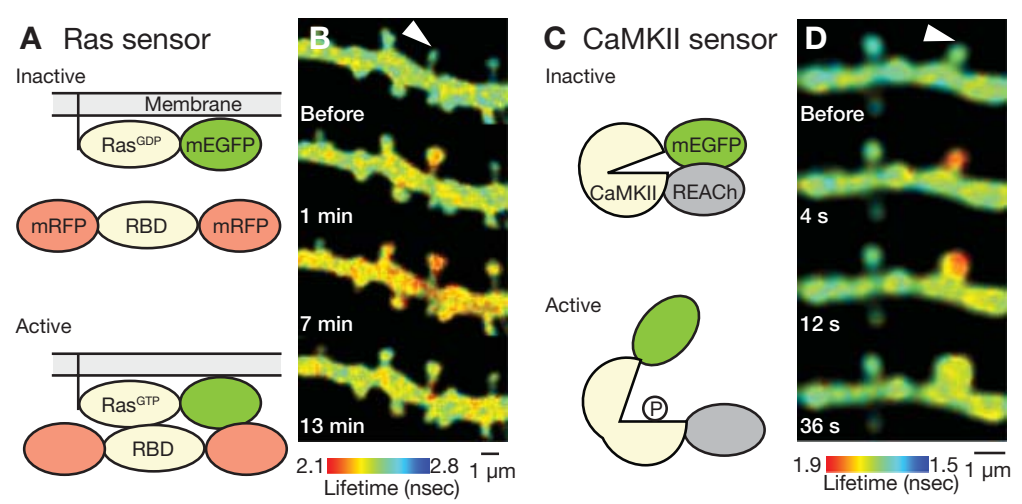

FIGURE 3. Signaling sensors designed for FLIM. (A) Schematic of the Ras activity sensor (Yasuda et al. 2006). Ras binds the Ras-binding domain (RBD) of cRaf1 when it is in the active form (GTP-bound form), causing FRET. (B) Fluorescence lifetime images of the Ras activity sensor within dendritic spines of pyramidal neurons in organotypic hippocampal slices. The spine marked with the arrowhead is stimulated with two-photon glutamate uncaging at time 0 (4 msec $\times$ $5 \mathrm{~mW}$ with a 720-nm Ti:sapphire laser, $0.5 \mathrm{~Hz}, 30$ times) in 2.5-mм MNI (4-methoxy-7-nitroindoline)-caged-L-glutamate in zero extracellular $\mathrm{Mg}^{++}$(Harvey et al. 2008). (C) Schematic of CaMKII activity sensor (Lee et al. 2009). CaMKII is in a closed conformation (causing FRET) when it is inactive, and opens on activation. Note that a decrease in FRET here denotes activity. $(D)$ Fluorescence lifetime images of the CaMKII activity sensor within dendritic spines of pyramidal neurons in organotypic hippocampus slices (Lee et al. 2009). The spine was stimulated with a protocol similar to that used in $(B)$. The fluorescence lifetime color map has a reversed orientation compared with $(B)$. 
Thus, to achieve an $\sim 3 \%$ noise level, $\sim 10^{3}$ photons per pixel are required. Thus, with a photoncounting rate of $10^{6} \mathrm{~Hz}$, it takes $\sim 1 \mathrm{msec}$ per pixel or $\sim 7 \mathrm{sec}$ per image with a $128 \times 128$-pixel resolution. Although this temporal resolution is sufficient for many signaling events (Fig. 3), if higher temporal resolution is needed, then the number of pixels must be reduced.

\section{OPTIMIZING SIGNALING SENSORS FOR FLIM}

Since the first FRET-based calcium sensors were developed (Miyawaki et al. 1997), a number of genetically encoded FRET sensors of signaling have been created (most of them use FRET between eCFP and eYFP). For example, a class of kinase activity reporters has been produced, which consist of four linked components: a donor, a specific kinase target polypeptide, a phosphoamino-acidbinding domain, and an acceptor ( $\mathrm{Ni}$ et al. 2006). Phosphorylation of the kinase target polypeptide causes binding between the kinase target polypeptide and the phosphoamino-acid-binding domain, thereby altering the FRET efficiency between the donor and the acceptor. Another class of kinase activity reporter uses fluorescent proteins tagged to both ends of the kinase to measure the conformational change of the kinase that occurs when the protein is activated (Takao et al. 2005; Ni et al. 2006). Most of these sensors are optimized for ratiometric imaging. Because the design criteria for ratiometric measurements and FLIM are different, the sensors must be reoptimized for FLIM (Yasuda 2006; Yasuda et al. 2006). Recently, sensors for Ras and CaMKII activity optimized for FLIM have been developed and were shown to be useful for measuring the activity of these proteins in single dendritic spines in hippocampal slices (Fig. 3). Ras activity was measured by quantifying the binding fraction between meGFP-Ras and Ras-binding domain of downstream protein tagged with two monomeric red fluorescent proteins at both ends (mRFP-RBD-mRFP) (Yasuda et al. 2006). When Ras is activated, mRFP-RBD-mRFP binds to meGFP-Ras, producing FRET between meGFP and mRFP (Fig. 3A,B). The CaMKII activity sensor measures the conformational change of CaMKII associated with the activation of CaMKII by meGFP and resonance energy-accepting chromoprotein (REACh) (nonradiative YFP variant) tagged to both ends of the molecule (Fig. 3C,D) (Lee et al. 2009). These sensors were originally made for the ratiometric method (Mochizuki et al. 2001; Takao et al. 2005) but further optimized for FLIM. To optimize sensors for FLIM, the following parameters can be modified.

Fluorophores

FLIM typically measures only the donor fluorescence; thus, the brightness and the photostability of the donor are important for a good signal-to-noise ratio, whereas the acceptor brightness is not. Also, monoexponential fluorescence decay of the donor is critical for measuring the fraction of donor bound to acceptor (Equations 2 and 3). eGFP and its monomeric mutants meGFP are optimal donors in FLIM because they are bright, are photostable, and have monoexponential fluorescence lifetime decay (Peter et al. 2005; Yasuda 2006; Yasuda et al. 2006).

mRFP and mCherry (a brighter variant of mRFP) (Shaner et al. 2004) have been used as acceptors in FLIM because of their good spectral separation from eGFP and meGFP (Peter et al. 2005; Yasuda 2006; Yasuda et al. 2006). The relatively low folding efficiency of mRFP and mCherry $(\sim 50 \%)$ decreases their sensitivity. To improve sensor sensitivity, two acceptors can be tagged to a protein. This increases the chance of having at least one mature acceptor (Yasuda et al. 2006) (Fig. 3A,B). The recently developed REACh (nonradiative YFP variant) and sREACh (a REACh variant with improved solubility, environmental insensitivity, and folding) are also reported to be useful as FLIM acceptors (Ganesan et al. 2006; Murakoshi et al. 2008). In particular, the high folding efficiency $(\sim 70 \%)$ of sREACh allows imaging with a high signal-to-noise ratio (Figs. 2 and $3 \mathrm{~B}$ ). REACh and sREACh, however, do not fluoresce; thus, their concentration cannot be measured easily. 
R. Yasuda

The eCFP-eYFP pair is the most popular for ratiometric imaging. However, this pair is not optimal for FLIM because eCFP is dim and has more than one fluorescence lifetime (Tramier et al. 2002; Yasuda et al. 2006), whereas FLIM does not take advantage of the brightness of eYFP.

\section{Location of Fluorophore and Linker}

Because FRET efficiency is sensitive to the distance and the angle between the two fluorophores, it depends on the length and the rigidity of the linkers connecting fluorophores and target proteins as well as the location of the fluorophore within the target protein (Miyawaki 2003). These parameters have to be optimized empirically (Miyawaki 2003). The optimum FRET efficiency is $\sim 50 \%$ for a protein-binding assay when using FLIM versus $100 \%$ for ratiometric imaging (Yasuda 2006; Yasuda et al. 2006).

\section{Binding Kinetics}

When using a sensor to observe interactions between a target protein and a binding partner that specifically binds to the active form of the protein (such as in a Ras sensor; Fig. 3A), the dissociation constants for binding between the two proteins significantly impact the sensitivity and kinetics of the sensor. The optimum dissociation constants are related to the expression level of the binding partner. For example, in the case of our Ras sensor, in which binding between meGFP-Ras and mRFP-RBDmRFP is used as a readout of Ras activity (Fig. 3A), a typical expression level of mRFP-RBD-mRFP in neurons is in the 1- to 20- $\mu \mathrm{M}$ range (H. Murakoshi and R. Yasuda, unpubl.). Thus, for optimal sensitivity, the dissociation constant between RBD and Ras ${ }^{\text {GTP }}$ (active form) must be lower than this range, and the dissociation between RBD and Ras ${ }^{\mathrm{GDP}}$ (inactive form) must be higher than these levels. Also, keep in mind that binding of the binding partner may slow down inactivation of the target protein. In the case of the Ras sensor (Fig. 3A), RBD binding to Ras ${ }^{\text {GTP }}$ competes with the activity of GTPase-activating proteins (GAPs) (Ras inactivator). More quantitatively, the inactivation time constant $\tau_{\mathrm{GAP}}$ is a function of [RBD] and $K_{\mathrm{D}}$,

$$
\tau_{\mathrm{GAP}}=1+\frac{[\mathrm{RBD}]}{K_{\mathrm{D}}} \tau_{\mathrm{GAP}}^{0}
$$

where $\tau_{\mathrm{GAP}}^{0}$ is $\tau_{\mathrm{GAP}}$ when no RBD is present and $K_{\mathrm{D}}$ is the dissociation constant of RBD and Ras ${ }^{\mathrm{GTP}}$ (Yasuda et al. 2006). To minimize slowdown of Ras inactivation by GAPs, $K_{\mathrm{D}}$ has to be higher than [RBD]. However, to obtain an optimal binding signal, $K_{\mathrm{D}}$ has to be lower than [RBD]. Thus, an RBD with a $K_{\mathrm{D}}$ similar to or slightly less than the expression level of RBD (micromolar level) should be chosen. The Ras sensor becomes almost irreversibly active when wild-type cRafl-RBD $\left(K_{\mathrm{D}} \sim 0.1 \mu \mathrm{M}\right)$ is used, whereas the sensor is inactivated as fast as endogenous Ras when a low-affinity mutant of RafRBD (R59A; $K_{\mathrm{D}} \sim 4 \mu \mathrm{M}$ ) is used (Yasuda et al. 2006; Harvey et al. 2008).

In cases in which the binding partner and the target protein are in the same polypeptide, such as in the ratiometric Ras sensor (Mochizuki et al. 2001), the optimal dissociation constant must be determined empirically (Ni et al. 2006).

One way to evaluate the dynamic range of a sensor is to coexpress the sensor with an activator or an inactivator protein within cells and to measure the sensor signals (Mochizuki et al. 2001). For some proteins, constitutively active or inactive mutants of the target protein could be used to test the dynamic range (Mochizuki et al. 2001; Yasuda et al. 2006). The dynamic range of FLIM sensors can be up to $\sim 50 \%$ in terms of the binding fraction or $\sim 25 \%$ in the mean fluorescence lifetime (Yasuda et al. 2006; Lee et al. 2009). 
Because the expression of a sensor can affect the kinetics of the sensor signal and can perturb cell signaling, it is necessary to evaluate the effects of overexpression on measured parameters such as time course and spatial spreading (Harvey et al. 2008). To do so, quantify the correlation between the parameters and the overexpression level, which is estimated from measurements of sensor concentration (Harvey et al. 2008). If a significant correlation is found, the value can be extrapolated to zero expression level to estimate the parameter values in the unperturbed system.

\section{Measurements of Sensor Concentration}

To measure the concentration of sensors transfected into cells, the fluorescence intensity of the sensor inside a compartment that is larger than the two-photon excitation volume within the cell (such as a primary dendrite in pyramidal neurons) can be measured with 2pLSM (Harvey et al. 2008; Lee et al. 2009). Next, the fluorescence intensity value is converted into concentration by comparing it with the fluorescence of a known concentration of purified fluorescent protein that has been measured separately (Lee et al. 2009). For cells deep within tissue, the effects of light scattering may have to be taken into account. To do so, fluorescent beads are injected near the cells of interest, and the measured fluorescence intensity of the beads is used to normalize the fluorescence intensity from the sensor (Gray et al. 2006; Harvey et al. 2008). That value is then converted into the fluorophore concentration by using the ratio between the fluorescence intensity of the beads in solution and the ratio of purified fluorescent protein with known concentration.

\section{CONCLUSION}

2pFLIM has proven to be useful for imaging signaling events in small subcellular compartments, especially in light-scattering tissue (Yasuda 2006; Harvey et al. 2008; Lee et al. 2009). The technique has been used to monitor several key proteins involved in synaptic plasticity, such as Ras (Yasuda 2006; Harvey et al. 2008) and CaMKII (Lee et al. 2009), within single dendritic spines in brain tissue. With continued optimization and development of FLIM sensors, the highly sensitive 2pFLIM will become increasingly applicable for imaging activity of a variety of proteins.

\section{ACKNOWLEDGMENTS}

I thank R. Kasliwal, M. Patterson, S.-J. Lee, and H. Murakoshi for discussions.

\section{REFERENCES}

Förster VT. 1993. Intermolecular energy migration and fluorescence (Translation of Förster T, 1948). In Biological physics (ed Mielczarek EV, et al.), pp. 183-221. American Institute of Physics, New York.

Ganesan S, Ameer-Beg SM, Ng TT, Vojnovic B, Wouters FS. 2006. A dark yellow fluorescent protein (YFP)-based resonance energy-accepting chromoprotein (REACh) for Förster resonance energy transfer with GFP. Proc Natl Acad Sci 103: 4089-4094

Gray NW, Weimer RM, Bureau I, Svoboda K. 2006. Rapid redistribution of synaptic PSD-95 in the neocortex in vivo. PLoS Biol 4: e370. doi: 10.1371/journal.pbio.0040370.

Harvey CD, Yasuda R, Zhong H, Svoboda K. 2008. The spread of Ras activity triggered by activation of a single dendritic spine. Science 321: 136-140.

Lakowicz JR. 2006. Principles of fluorescence spectroscopy, 3rd ed. Plenum, New York.
Lee SJ, Escobedo-Lozoya Y, Szatmari EM, Yasuda R. 2009. Activation of CaMKII in single dendritic spines during long-term potentiation. Nature 458: 299-304.

Miyawaki A. 2003. Visualization of the spatial and temporal dynamics of intracellular signaling. Dev Cell 4: 295-305.

Miyawaki A, Llopis J, Heim R, McCaffery JM, Adams JA, Ikura M, Tsien RY. 1997. Fluorescence indicators for $\mathrm{Ca}^{2+}$ based on green fluorescent proteins and calmodulin. Nature 388: 882-887.

Mochizuki N, Yamashita S, Kurokawa K, Ohba Y, Nagai T, Miyawaki A, Matsuda M. 2001. Spatio-temporal images of growth-factor-induced activation of Ras and Rap1. Nature 411: 1065-1068.

Murakoshi H, Iino R, Kobayashi T, Fujiwara T, Ohshima C, Yoshimura A, Kusumi A. 2004. Single-molecule imaging analysis of Ras activation in living cells. Proc Natl Acad Sci 101: 7317-7322. 
Murakoshi H, Lee S-J, Yasuda R. 2008. Highly sensitive and quantitative FRET-FLIM imaging in single dendritic spines using improved nonradiative YFP. Brain Cell Biol 36: 31-42.

Ni Q, Titov DV, Zhang J. 2006. Analyzing protein kinase dynamics in living cells with FRET reporters. Methods 40: 279-286.

Peter M, Ameer-Beg SM, Hughes MK, Keppler MD, Prag S, Marsh M, Vojnovic B, Ng T. 2005. Multiphoton-FLIM quantification of the EGFP-mRFP1 FRET pair for localization of membrane receptorkinase interactions. Biophys J 88: 1224-1237.

Philip J, Carlsson K. 2003. Theoretical investigation of the signal-to-noise ratio in fluorescence lifetime imaging. J Opt Soc Am A 20: 368-379.

Shaner NC, Campbell RE, Steinbach PA, Giepmans BN, Palmer AE, Tsien RY. 2004. Improved monomeric red, orange and yellow fluorescent proteins derived from Discosoma sp. red fluorescent protein. Nat Biotechnol 22: 1567-1572.

Takao K, Okamoto K, Nakagawa T, Neve RL, Nagai T, Miyawaki A, Hashikawa T, Kobayashi S, Hayashi Y. 2005. Visualization of synaptic
$\mathrm{Ca}^{2+} /$ calmodulin-dependent protein kinase II activity in living neurons. J Neurosci 25: 3107-3112.

Tramier M, Gautier I, Piolot T, Ravalet S, Kemnitz K, Coppey J, Durieux C, Mignotte V, Coppey-Moisan M. 2002. Picosecond-hetero-FRET microscopy to probe protein-protein interactions in live cells. Biophys J 83: 3570-3577.

Wallrabe H, Periasamy A. 2005. Imaging protein molecules using FRET and FLIM microscopy. Curr Opin Biotechnol 16: 19-27.

Yasuda R. 2006. Imaging spatiotemporal dynamics of neuronal signaling using fluorescence resonance energy transfer and fluorescence lifetime imaging microscopy. Curr Opin Neurobiol 16: 551-561.

Yasuda R, Harvey CD, Zhong H, Sobczyk A, van Aelst L, Svoboda K. 2006. Super-sensitive Ras activation in dendrites and spines revealed by twophoton fluorescence lifetime imaging. Nat Neurosci 9: 283-291.

Zacharias DA, Violin JD, Newton AC, Tsien RY. 2002. Partitioning of lipidmodified monomeric GFPs into membrane microdomains of live cells. Science 296: 913-916. 


\section{Imaging Intracellular Signaling Using Two-Photon Fluorescent Lifetime Imaging Microscopy}

Ryohei Yasuda

Cold Spring Harb Protoc; doi: 10.1101/pdb.top072090

\begin{tabular}{|c|c|}
\hline $\begin{array}{r}\text { Email Alerting } \\
\text { Service }\end{array}$ & Receive free email alerts when new articles cite this article - click here. \\
\hline $\begin{array}{r}\text { Subject } \\
\text { Categories }\end{array}$ & $\begin{array}{l}\text { Browse articles on similar topics from Cold Spring Harbor Protocols. } \\
\text { Cell Biology, general (1382 articles) } \\
\text { Cell Imaging (525 articles) } \\
\text { Fluorescence (517 articles) } \\
\text { Fluorescent Proteins ( } 259 \text { articles) } \\
\text { Imaging of Protein: Protein Interactions (22 articles) } \\
\text { Multi-Photon Microscopy (103 articles) } \\
\text { Protein: Protein Interactions ( } 86 \text { articles) } \\
\text { Visualization (524 articles) } \\
\text { Visualization of Proteins (107 articles) }\end{array}$ \\
\hline
\end{tabular}

